\title{
Terahertz diffraction enhanced transparency probed in the near field
}

\author{
Alexei Halpin, ${ }^{1}$ Niels van Hoof, ${ }^{1}$ Arkabrata Bhattacharya, ${ }^{1}$ Christiaan Mennes, ${ }^{2}$ and Jaime Gomez Rivas ${ }^{1,3}$ \\ ${ }^{1}$ DIFFER - Dutch Institute for Fundamental Energy Research, De Zaale 20, Eindhoven, Netherlands \\ ${ }^{2}$ Centre for Nanophotonics, AMOLF, Science Park 104, Amsterdam, Netherlands \\ ${ }^{3}$ Department of Applied Physics and Institute for Photonic Integration Eindhoven University of Technology \\ P.O. Box 5135600 MB Eindhoven - The Netherlands
}

(Received 11 April 2017; revised manuscript received 22 June 2017; published 8 August 2017)

\begin{abstract}
Electromagnetically induced transparency in metamaterials allows to engineer structures which transmit narrow spectral ranges of radiation while exhibiting a large group index. Implementation of this phenomenon frequently calls for strong near-field coupling of bright (dipolar) resonances to dark (multipolar) resonances in the metamolecules comprising the metamaterials. The sharpness and contrast of the resulting transparency windows thus depends strongly on how closely these metamolecules can be placed to one another, placing constraints on fabrication capabilities. In this manuscript, we demonstrate that the reliance on near-field interaction strength can be relaxed, and the magnitude of the electromagnetic-induced transparency enhanced, by exploiting the long-range coupling between metamolecules in periodic lattices. By placing dolmen structures resonant at $\mathrm{THz}$ frequencies in a periodic lattice, we show a significant increase of the transparency window when the in-plane diffraction is tuned to the resonant frequency of the metamolecules, as confirmed by direct mapping of the $\mathrm{THz}$ near-field amplitude across a lattice of dolmens. Through the direct interrogation of the dark resonance in the near field, we show the interplay of near- and far-field couplings in optimizing the response of planar dolmen arrays via diffraction-enhanced transparency.
\end{abstract}

DOI: 10.1103/PhysRevB.96.085110

\section{INTRODUCTION}

Resonant metallic structures can be engineered to possess very high scattering cross sections, and constitute versatile components for the manipulation of electromagnetic radiation. They are frequently placed in densely packed arrays, which then demonstrate the response of the constituent elements over larger areas. In the limit of high densities, i.e., when the average distance between scatterers becomes smaller than the wavelength of radiation, we reach the regime of metasurfaces or metamaterials [1,2], where the scatterers represent the constituent metamolecules. In metamaterials, the response to electromagnetic radiation is then approximated by an effective medium with unconventional or exotic properties [3].

One such property that has garnered attention is plasmonic electromagnetically induced transparency (EIT) [4-7]. In plasmonic EIT, a medium can be rendered transparent to incident radiation over a narrow spectral range due to the near-field coupling between bright (dipolarlike) plasmonic resonances and dark (multipolar) resonances supported by metallic scatterers. This coupling and interference of resonances gives rise to a large far-field transmission at frequencies of high near-field enhancement, which significantly increases the group index in the material and thus reduces the group velocity of the radiation traversing it. As a result, EIT offers opportunities for the manipulation of electromagnetic radiation for optical communication applications and sensing using thin planar arrays of scatterers. One important disadvantage of plasmonic EIT, however, is its reliance on near-field coupling between resonators $[4,8]$. This requires precise positioning of the structures to create small gaps between them, unless the structures are arranged in three dimensions [6,9], defining fabrication constraints which limit the magnitude of the induced transparency.

In this manuscript, we exploit the long-range coupling between metamolecules in a periodic lattice to demonstrate an enhanced transparency at terahertz $(\mathrm{THz})$ frequencies that can be termed diffraction-enhanced transparency (DET). The metamolecules in this case are dolmen structures consisting of coupled resonators: a dipolar rod separated by a small gap from a pair of orthogonally oriented rods with quadrupolar character. The dolmens are arranged in a periodic lattice, where the response to the incident electromagnetic radiation is enhanced by diffraction. Recently, the combination of radiative and near-field couplings has drawn attention in this respect, for demonstrating DET in split-ring resonator (SRR) arrays [10], and pairs of detuned dipoles [11], as well as for exciting radiant antisymmetric collective resonances in near-field coupled structures [12].

Here we exploit near-field microscopy at $\mathrm{THz}$ frequencies, a powerful technique which allows for the measurement of the electric near field in both amplitude and phase with subwavelength resolutions, to experimentally demonstrate the dynamics underlying DET. In this way we measure the exchange of energy between the bright and dark resonators that leads to DET by directly probing the near field in the vicinity of the individual bright and dark resonators in the lattice. As a result, we show how the appropriate tuning of the lattice allows for the efficient excitation of the subradiant mode in the dolmens as compared to the isolated structures. A sharper and deeper transparency window is thus achieved without varying the intradolmen coupling, relaxing the requirement for very small gaps and strong near-field coupling between the constituent elements of the dolmen.

The dolmen structure is one of many geometries giving rise to EIT [4,7,13-15]. It consists of two elements: a metallic rod oriented along the polarization of the incident field, and two orthogonally oriented rods placed nearby each end of the first rod. The top rod is tuned to support a dipolarlike resonance resulting from the driven oscillation of the charges in the metal, providing a radiant mode which can couple to 
(a)

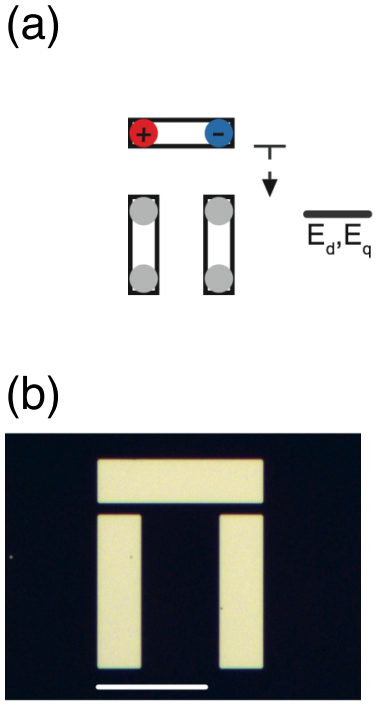

(b)

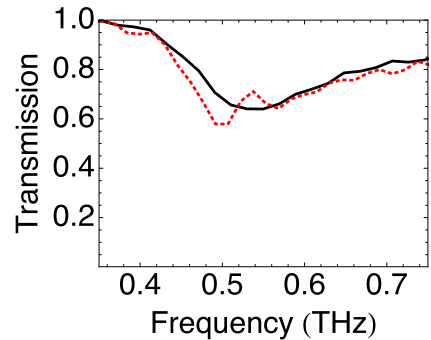

(c)

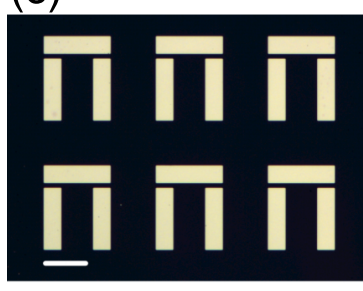

Frequency $(\mathrm{THz})$ (e)

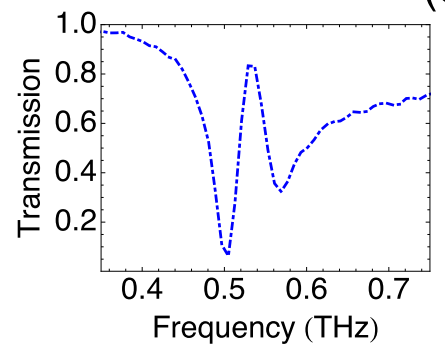

FIG. 1. (a) Illustration of near-field coupling in dolmen structures. Dipolar $\left(\mathrm{E}_{d}\right)$ and quadrupolar $\left(\mathrm{E}_{q}\right)$ resonances in metallic rods interact in the near field as the rods are brought together, leading to bonding $\left(\mathrm{E}_{b}\right)$ and antibonding $\left(\mathrm{E}_{a b}\right)$ resonances and resulting in the appearance of a transparency window. (b) Optical microscope image of a dolmen structure, scale bars of $100 \mu \mathrm{m}$ shown in white. (c) Microscope image of the dolmen lattice with pitches $\mathrm{p}_{x}=250 \mu \mathrm{m}$ and $\mathrm{p}_{y}=290 \mu \mathrm{m}$. (d) Intensity transmission spectrum of a random array of dolmens with a $12 \%$ filling fraction (dotted red) and transmission spectrum of dipolar resonators with 5\% (solid black). (e) Transmission spectrum of the dolmen lattice.

incident radiation. The vertical rod pair supports a subradiant quadrupolar mode, however, which cannot be directly excited by incident radiation due to symmetry. The radiant mode is spectrally broad due to its strong radiative losses, while the subradiant mode is spectrally narrow. If the distance between the two elements of the dolmen is sufficiently small, the near field of the top rod will couple to the orthogonal rods and drive the subradiant mode in a manner which can be well described by a classical coupled oscillator model $[3,16]$.

The consequences of this near-field coupling are thus the excitation of a quadrupolar mode leading to a transparency window in the far-field spectrum, and the associated formation of resonances which hybridize the constituent dipolar and quadrupolar modes. These hybrid modes are commonly referred to as bonding and antibonding modes on the basis of the relative phase of the polarization, as illustrated in Fig. 1(a). For this nonradiative near-field coupling, the interaction occurs on a subwavelength length scale via the evanescent near fields of the dolmen's elements. Therefore, very small gaps between the elements are appealing due to the enhanced coupling strength between the resonators $[4,6,17]$, placing a strict restriction on fabrication. As we show ahead, this condition can be relaxed by exploiting the long-range coupling of dolmens in a lattice.

\section{MATERIALS AND METHODS}

The investigated dolmen structures consist of gold $(\mathrm{Au})$ evaporated onto patterned photoresist by UV lithography using 3-mm-thick quartz as a substrate. The dipolar rods have a length of $150 \mu \mathrm{m}$ and a width of $40 \mu \mathrm{m}$, while the quadrupolar rods have dimensions of $140 \mu \mathrm{m}$ by $40 \mu \mathrm{m}$, as can be seen in the microscope image of Fig. 1(b). All structures have a gold thickness of $100 \mathrm{~nm}$. The spacing between the vertical rods is set to $80 \mu \mathrm{m}$, and the distance between the top dipolar resonator and the vertical quadrupolar rod pair is $10 \mu \mathrm{m}$. In Fig. 1(c), we show a microscope image of the periodic sample with a pitch of $\mathrm{p}_{x}=250 \mu \mathrm{m}$ and $\mathrm{p}_{y}=290 \mu \mathrm{m}$. In order to facilitate the comparison of the intrinsic far-field response of the dolmens to that of the periodic samples, we also prepared samples where the dolmens are placed in a random array with a filling fraction of $12 \%$, where the filling fraction is defined by the total area covered by metal. The far-field transmission spectra of the samples were measured using a $\mathrm{THz}$ time-domain spectrometer consisting of a photoconductive antenna and photoconductive switch separated by lenses in a 4- $f$ configuration (Menlo Systems $\mathrm{GmbH}$, Germany). The transmission measurements are referenced to a quartz substrate. The $\mathrm{THz}$ near-field microscope used in these measurements has been described previously [18], where THz microprobes [19] (Protemics GmbH, Germany) are used to measure the near field at a distance of $0.5 \mu \mathrm{m}$ above the sample. The THz microprobes used in these experiments allow isolation of a specific polarization of the electric near field. The spatially resolved near-field temporal dynamics are collected by raster scanning the sample through the optical axis defined by the incident beam and the near-field tip, where Fourier analysis of the obtained $\mathrm{THz}$ transients allows the subsequent generation of $2 \mathrm{D}$ spectral maps.

\section{RESULTS AND DISCUSSION}

The far-field transmission spectra for the dolmens outside of the lattice exhibit a two-dip structure with a small peak of increased transmission between them, as seen in Fig. 1(d). The frequency of this transmission peak $(v=$ $0.53 \mathrm{THz})$ corresponds to the resonance frequency of the dipolar resonator [shown in solid black in Fig. 1(d)]. The two dips in the dolmen's far-field transmission are associated with the so-called bonding and antibonding modes in the structure [4], surrounding the transparency window. This transparency window is very modest for dolmens with a gap size distance of $10 \mu \mathrm{m}$ separating the top dipolar resonator and the vertical quadrupolar resonator. This indicates that the near-field coupling between bright and dark resonators would need to be increased in order to generate significant EIT for the isolated dolmen structure [20].

However, by using the same dolmen as the metamolecule for a periodic array, a drastic difference is immediately borne 


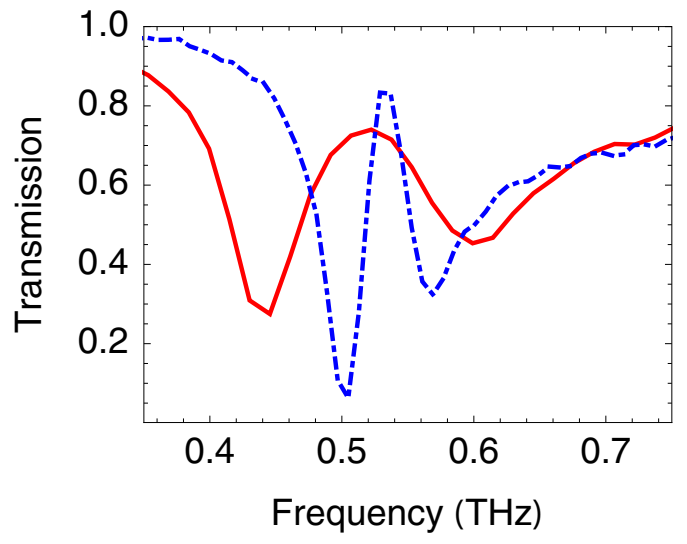

FIG. 2. Far-field transmission spectra corresponding to a random array of dolmen structures with a gap size of $1 \mu \mathrm{m}$ (solid red), as compared to the spectrum for dolmen structures with a $10 \mu \mathrm{m}$ gap size but placed in a periodic lattice (dashed blue).

out in the far-field extinction in Fig. 1(e). There we find a more pronounced transparency window, and sharper peaks at the spectral locations of the bonding $(v=0.51 \mathrm{THz})$ and antibonding $(v=0.58 \mathrm{THz})$ modes. In this lattice, we select the pitch along the $y$-direction $\left(\mathrm{p}_{y}\right)$ to correspond to the Rayleigh anomaly (RA) condition for normal incidence in glass at the frequency of the dipolar resonance, namely

$$
\lambda_{R A}=n p_{y},
$$

where $n$ is the refractive index of the substrate. The RA condition corresponds to the frequency at which incident radiation normal to the array is diffracted into the plane of the array. Therefore, the incident $\mathrm{THz}$ field that excites the bright resonance not only couples to the quadrupolar resonance through the near field, but also promotes radiative coupling between the resonators.

In Fig. 2, we demonstrate for comparison the case of structures where the intradolmen coupling is increased by reducing the gap separating the horizontal and vertical rods. For this case we observe that, for a $1 \mu \mathrm{m}$ gap separation, the transparency window is well resolved due to a stronger interaction between the constituent bright and dark resonators, yet the spectral range over which the transparency occurs is broader, owing to the greater separation between bonding and antibonding modes. This broad transparency can be undesirable for certain applications, since the anomalous dispersion accompanying EIT will be strongest for sharp features due to the Kramers-Kronig relations. Moreover, further increasing the transparency requires yet smaller gaps, which can become susceptible to fabrication errors. The periodic sample, however, exhibits a sharp and deep transparency window, which is more appealing for maximizing the group index, and is resilient against imperfections in sample preparation.

We have performed $\mathrm{THz}$ near-field measurements to further investigate the effect of the lattice in this system, and to characterize the electromagnetic coupling between the different elements. Near-field $\mathrm{THz}$ microscopy has seen tremendous progress over recent years towards very high spatial resolutions [21-24], and has previously been employed (a)
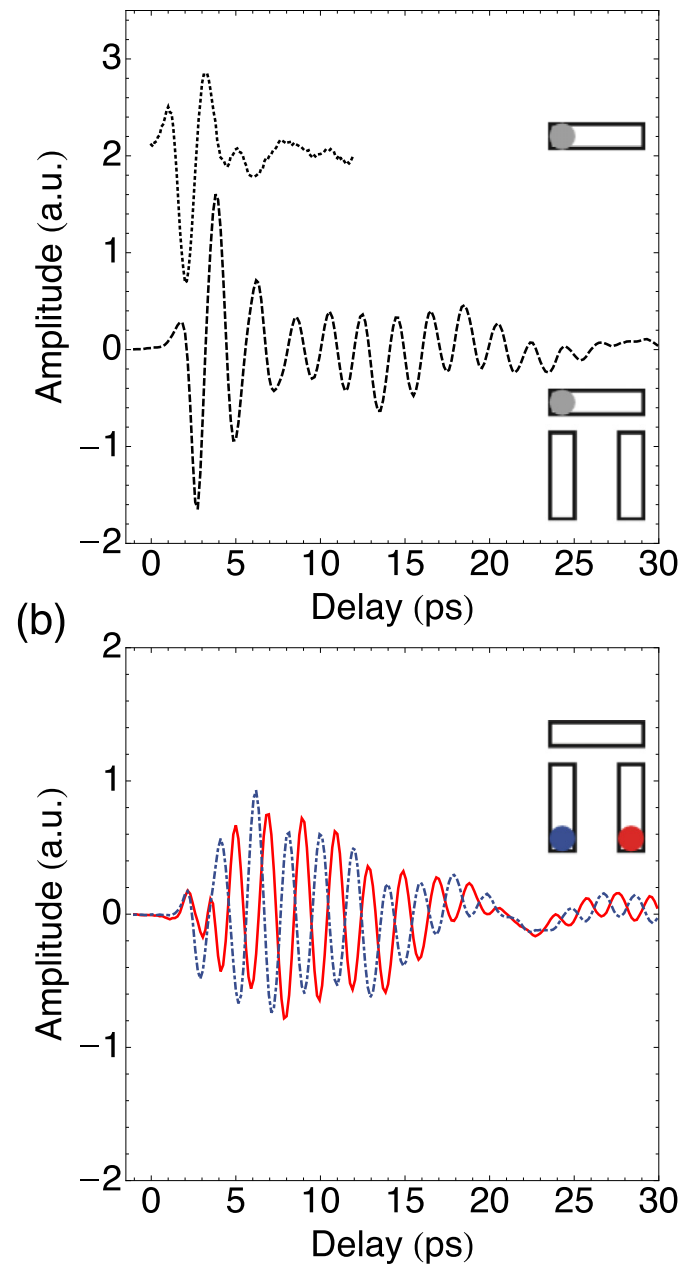

FIG. 3. (a) THz transients of the out-of-plane component of the electric near field at the tip of the dipolar resonator in the dolmen in a periodic lattice (dashed). For reference, the fields induced in an isolated rod are also shown (dotted). (b) THz transients corresponding to the near field measured at the tips of quadrupolar rod pair (solid red and dot-dashed blue). The insets indicate (circles) the positions on the dolmens at which the transients were measured.

in many successful implementations to explore near-field dynamics in both resonant structures $[18,25,26]$ and apertures [27-31]. In the work of Bitzer et al. [25], lattice modes were investigated for the case of split ring resonators (SRR), where the array was shown to hybridize lattice modes with magnetic dipole resonances. The lattice geometry was thus used to redistribute electric fields in the vicinity of the SRR and modify the quality factors of the modes by suppressing their radiative losses.

In Fig. 3, we show the experimental THz time domain amplitude of the $\mathrm{E}_{z}$ (out-of-plane) component of the field, corresponding to near-field transients recorded at different spatial locations on the sample. For reference, we also show the near-field transient collected at the end of an isolated dipolar rod in Fig. 3(a), where we observe damped oscillations corresponding to the dipolar resonance excited in the rod, with a rapidly decaying amplitude due to aforementioned radiative 


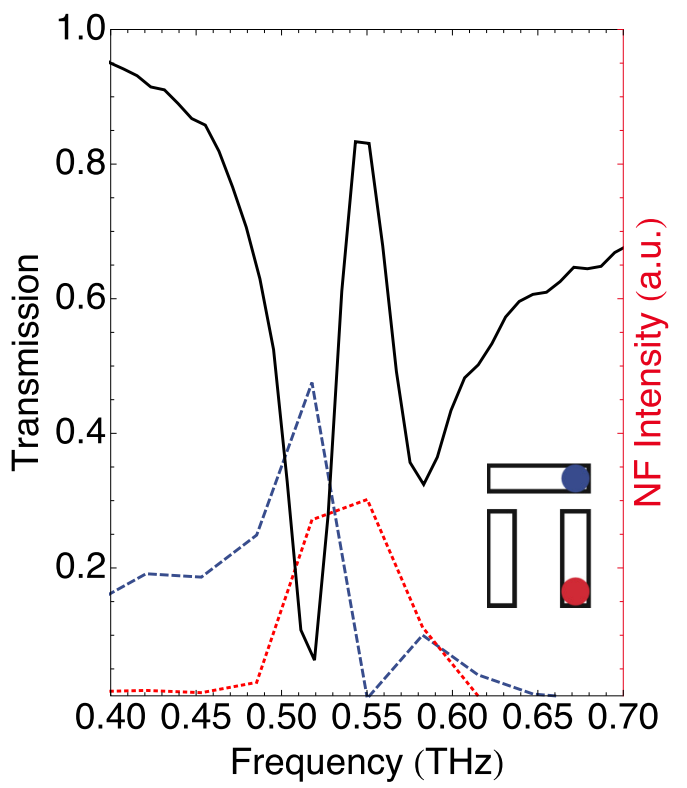

FIG. 4. Far-field transmission spectrum of dolmen lattice (solid black), with power spectra of the near-field transients measured at the end of vertical rod (dotted red), and at the end of the horizontal rod (dashed blue). The inset indicates the positions (circles) on the dolmen at which the near-field power spectra were measured, revealing the excitation of the dark quadrupolar mode.

losses. When probing the same position at the end of the dipolar rod in the dolmen, we observe a markedly different transient. In particular, we measure underdamped oscillations persisting for over $20 \mathrm{ps}$ following excitation, which illustrates the enhanced group index caused by DIT. These oscillatory components are also manifest when probing the ends of the vertical rod pair, where we record an opposite phase in the $\mathrm{THz}$ transient amplitude oscillation measured on either side of the pair, as seen in Fig. 3(b). These correspond to the out-of-phase oscillations of the fundamental resonances in each vertical rod, defining the overall quadrupolar character for the subradiant mode in the rod pair. In Fig. 4, we show a comparison of the transmission spectra measured in the far-field (black solid curve) and the near-field spectra measured in the vicinity of the quadrupolar rod (red dotted curve) and dipolar rod (blue dashed curve). These near-field spectra correspond to the power spectra of the Fourier-transformed transients in Fig. 3. These results demonstrate how the transparency window in the far field, with a transmission higher than $80 \%$, occurs at the resonant frequency of the quadrupolar dark mode, showing the origin of the increased transparency in the coupling to this dark mode. Likewise, the near-field spectrum corresponding to the horizontal rod matches that which is radiated to the far field and observed in the transmission spectrum. To uncover the electric field distributions responsible for the features in the far-field transmission shown in Fig. 4, we display 2D maps of the real part of the $\mathrm{E}_{z}$ field component at three different frequencies in Fig. 5, obtained by Fourier transformation of the transients measured across three unit cells in the lattice. Notably, the near-field maps reveal the hybridization of bright and dark modes resulting from near-field coupling in the dolmen. The bonding mode occurring at $v=0.51 \mathrm{THz}$ can (a)

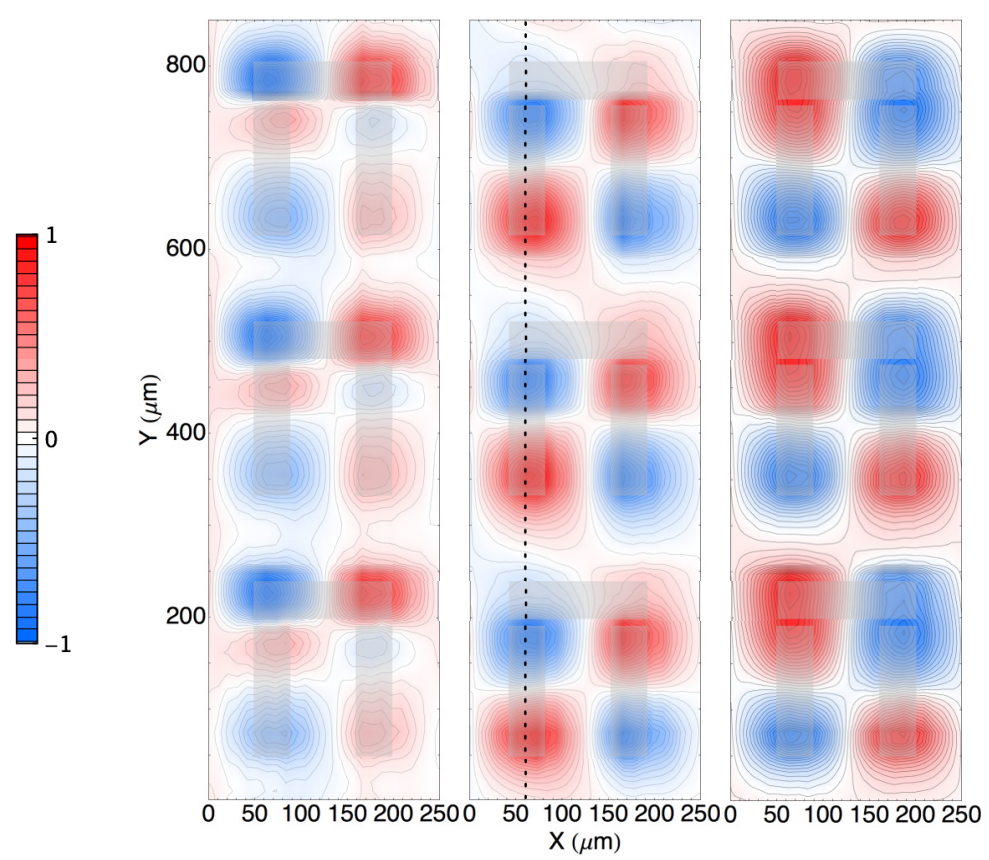

(d)

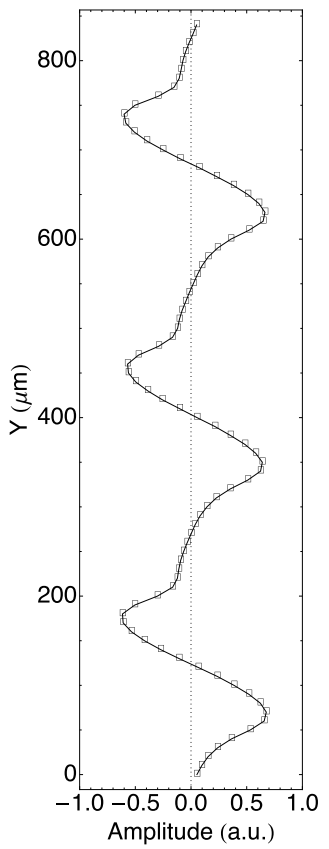

FIG. 5. (a) Near-field map corresponding to the $\mathrm{E}_{z}$ component at a frequency of $v=0.51 \mathrm{THz}$, showing the bonding mode resulting from the near-field coupling between bright and dark resonators in a lattice of dolmens. The maps show three unit cells along $y$. (b) Near-field map corresponding to the $\mathrm{E}_{z}$ component at a frequency of $v=0.55 \mathrm{THz}$, corresponding to the DIT frequency. (c) Near-field map of the $\mathrm{E}_{z}$ component at a frequency of $v=0.58 \mathrm{THz}$, showing the antibonding mode. (d) Cross section through panel (b) along the dotted line showing the extended fields associated with radiative coupling across the y-direction. All maps are normalized to their respective maxima. 
be seen in Fig. 5(a), and is associated with a superposition of dipolar and quadrupolar modes. Here the relative phase between these modes leads to an attractive electrostatic interaction across the gap of the dolmens, lowering the bonding mode energy relative to the bare dipolar resonance. Likewise, in Fig. 5(c) we observe the antibonding mode at a frequency of $v=0.58 \mathrm{THz}$, where the phase between the dipolar and quadrupolar resonances is reversed, leading to repulsive interactions and a higher energy for that mode. In Fig. 5(b), we observe that the near fields at the frequency $v=0.55 \mathrm{THz}$ are enhanced in the vicinity of the quadrupolar rod pair, revealing the excitation of the dark mode in the lattice giving rise to the DET window in the transmission spectrum of Fig. 4.

Moreover, for all near-field maps in Fig. 5, we observe fields extending across the unit cell, which highlights the lattice-assisted coupling. This field extension across unit cells is best visualized in Fig. 5(d), which displays the $\mathrm{E}_{z}$ field amplitude at $v=0.55 \mathrm{THz}$ along the dotted line shown in Fig. 5(b). We should stress, however, that we are not yet in the near-field coupling regime across the unit cell. For the lattices studied here, with a pitch along the y-direction of $290 \mu \mathrm{m}$, the RA occurs at the same frequency as both the bright and dark intradolmen modes. As the incident field drives a polarization along the length $(\mathrm{x})$ of the dipolar rods, the radiation patterns from the rods will be strongest along the orthogonal direction (y) at the RA condition [32].

This efficient radiative coupling between the dipolar resonances of scatterers and surface modes has previously been shown to result in strong high-Q resonances with Fanolike lineshapes [33-35] in arrays of dipolar resonators at optical frequencies, and has also been exploited for reducing radiative losses in arrays of scatterers [25]. In the system studied here, the losses of the dipolar mode are reduced by the same mechanism, leading to a higher quality factor and thus allowing for more cycles of exchange between the radiant and subradiant modes before the decay of the polarization. As a result, we observe an improved coupling of the incident field to the quadrupolar mode and pronounced DET in the far field. To further illustrate how the lattice-enhanced coupling can be used to optimize EIT in arrays of dolmens, we have performed full-wave simulations using the Finite Difference Time Domain (FDTD) method to explore the effect of $\mathrm{p}_{y}$ on the transmission spectrum. These results are shown in Fig. 6, where we demonstrate the difference between dolmens possessing a gap of $10 \mu \mathrm{m}$, for several values of $\mathrm{p}_{y}$ spanning both the metamaterial and radiative coupling limits. For very small pitches on the order of $220 \mu \mathrm{m}$ or less, we observe a single broad peak in the transmission spectrum and no transparency window.

For those small periodicities, the detuning between the RA $(v \sim 0.65 \mathrm{THz})$ and the dipolar mode is large, to the detriment of radiative coupling. In this limit, near-field coupling also occurs between dolmens in adjacent unit cells, such that they no longer act as independent metamolecules. The associated breakdown of the tight-binding approximation results in new resonances which can no longer be understood by "bonding" or "antibonding" modes as we have described here, since the dipolar rods couple capacitively both to the vertical rod pairs below and above them. Here, what we previously referred

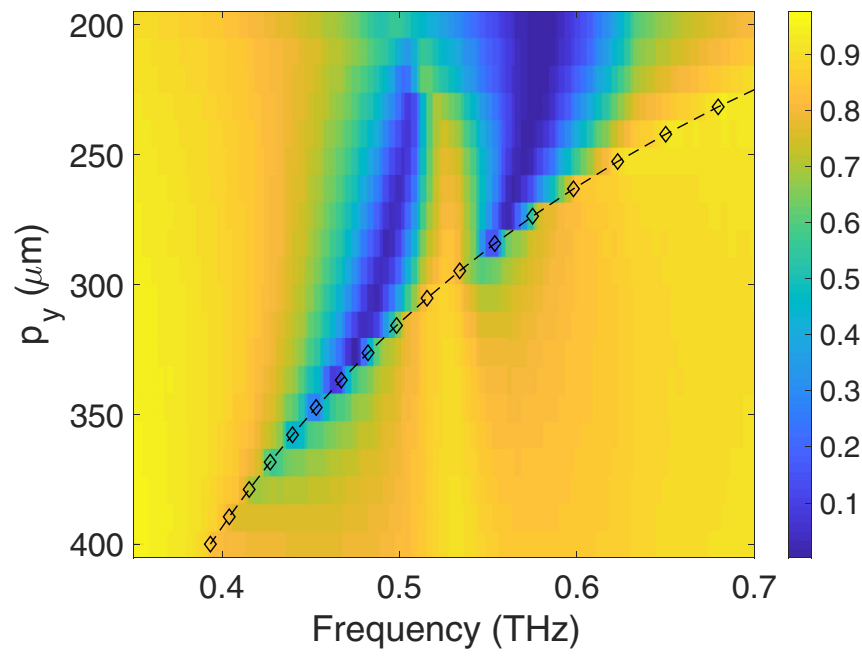

FIG. 6. Numerically calculated far-field transmission spectra for a fixed $\mathrm{p}_{x}=250 \mu \mathrm{m}$, for values of $\mathrm{p}_{y}$ spanning both the radiative coupling and metamaterial limits. The simulations are performed using commercial FDTD software. The Rayleigh anomaly condition for each pitch is plotted by dashed line.

to as the bonding mode becomes strongly suppressed due to symmetry reasons, as has been recently described in detail for dolmens at near-infrared frequencies [36].

On the other hand, as we approach the overlap of the RA condition with the dipolar and quadrupolar modes in the system, the transparency window reaches its maximal value, owing to the interplay between the three coupled modes in the system. Here, the interference between the RA, radiant, and subradiant modes yields sharp features in the transmission spectra due to the suppression of radiative losses, where the precise detuning condition allows to control the intensities of bonding and antibonding modes. For larger values of pitch, we reach a regime where neither near-field nor radiative couplings contribute, and where the lower density of scatterers leads to weak extinction of the incident field and the absence of EIT.

\section{CONCLUSIONS}

In conclusion, the combination of near-field and radiative couplings can be used to significantly enhance the EIT in coupled resonant systems. We have probed this phenomenon using near-field $\mathrm{THz}$ microscopy to selectively interrogate the constituent resonators in the dolmen. This near-field study reveals the hybridization of bright and dark modes in resonant structures when placed in a lattice where the additional coupling to in-plane diffractive orders occurs. Making use of DET also relaxes constraints placed on the distance between elements in coupled resonant structures, which may be very practical for the extension of DET to previously investigated plasmonic structures in other spectral regions where fabrication is more challenging. We expect that extensions on this principle could be readily harnessed both for slow-light applications and strong light-matter coupling. 


\section{ACKNOWLEDGMENTS}

The authors acknowledge Mohammad Ramezani and A. F. Koenderink for helpful discussions. The authors additionally acknowledge Niels Commandeur and Luc Blom for technical support. We would like to acknowledge financial support through ERC Starting Grant No. 259272 THZ-PLASMON (2010), and ERC Proof of Concept Grant No. 665619 MicroMap (2014).
[1] J. B. Pendry, D. Schurig, and D. R. Smith, Science 312, 1780 (2006).

[2] N. Yu, P. Genevet, M. A. Kats, F. Aieta, J.-P. Tetienne, F. Capasso, and Z. Gaburro, Science 334, 333 (2011).

[3] P. Tassin, L. Zhang, T. Koschny, E. N. Economou, and C. M. Soukoulis, Phys. Rev. Lett. 102, 053901 (2009).

[4] S. Zhang, D. A. Genov, Y. Wang, M. Liu, and X. Zhang, Phys. Rev. Lett. 101, 047401 (2008).

[5] N. Papasimakis, V. A. Fedotov, N. I. Zheludev, and S. L. Prosvirnin, Phys. Rev. Lett. 101, 253903 (2008).

[6] N. Liu, L. Langguth, T. Weiss, J. Kästel, M. Fleischhauer, T. Pfau, and H. Giessen, Nat. Mater. 8, 758 (2009).

[7] Z. Zhu, X. Yang, J. Gu, J. Jiang, W. Yue, Z. Tian, M. Tonouchi, J. Han, and W. Zhang, Nanotech. 24, 214003 (2013).

[8] T. Coenen, D. T. Schoen, B. J. M. Brenny, A. Polman, and M. L. Brongersma, Phys. Rev. B 93, 195429 (2016).

[9] Y. Todorov, P. Desfonds, C. Belacel, L. Becerra, and C. Sirtori, Opt. Express 23, 16838 (2015).

[10] M. Manjappa, Y. K. Srivastava, and R. Singh, Phys. Rev. B 94, 161103 (2016).

[11] M. C. Schaafsma, A. Bhattacharya, and J. G. Rivas, ACS Photon. 3, 1596 (2016).

[12] A. D. Humphrey, N. Meinzer, T. A. Starkey, and W. L. Barnes, ACS Photon. 3, 634 (2016).

[13] N. Li, X. Tian, W. Zhang, L. Luo, G. Li, and Z. Zhang, Sens. Actuators, A (Physical) 234, 346 (2015).

[14] H.-m. Li, S.-b. Liu, S.-y. Wang, S.-y. Liu, Y. Hu, and H.-b. Li, Sci. Reports 6, 21457 (2016).

[15] S. Panaro, F. De Angelis, and A. Toma, Opt. Lasers Eng. 76, 64 (2016).

[16] C. L. G. Alzar, M. A. G. Martinez, and P. Nussenzveig, Am. J. Phys. 70, 37 (2002).

[17] N. Liu, T. Weiss, M. Mesch, L. Langguth, U. Eigenthaler, M. Hirscher, C. Sönnichsen, and H. Giessen, Nano Lett. 10, 1103 (2010).
[18] A. Bhattacharya, G. Georgiou, S. Sawallich, C. Matheisen, M. Nagel, and J. Gómez Rivas, Phys. Rev. B 93, 035438 (2016).

[19] M. Wächter, M. Nagel, and H. Kurz, Appl. Phys. Lett. 95, 041112 (2009).

[20] A. Halpin, C. Mennes, and A. Bhattacharya, Appl. Phys. Lett. 110, 101105 (2017).

[21] R. I. Stantchev, B. Sun, S. M. Hornett, P. A. Hobson, G. M. Gibson, M. J. Padgett, and E. Hendry, Sci. Adv. 2, e1600190 (2016).

[22] F. Kuschewski, H. G. von Ribbeck, J. Döring, S. Winnerl, L. M. Eng, and S. C. Kehr, Appl. Phys. Lett. 108, 113102 (2016).

[23] F. Blanchard and K. Tanaka, Opt. Lett. 41, 4645 (2016).

[24] A. Ryabov and P. Baum, Science 353, 374 (2016).

[25] A. Bitzer, J. Wallauer, H. Helm, H. Merbold, T. Feurer, and M. Walther, Opt. Express 17, 22108 (2009).

[26] A. Bitzer, A. Ortner, H. Merbold, and T. Feurer, Opt. Express 19, 2537 (2011).

[27] M. A. Seo, A. J. L. Adam, J. H. Kang, J. W. Lee, S. C. Jeoung, Q. H. Park, P. C. M. Planken, and D. S. Kim, Opt. Express 15, 11781 (2007).

[28] R. Chakkittakandy, J. A. Corver, and P. C. Planken, Opt. Express 16, 12794 (2008).

[29] A. Bitzer and M. Walther, Appl. Phys. Lett. 92, 231101 (2008).

[30] M. A. Seo, H. R. Park, S. M. Koo, D. J. Park, J. H. Kang, O. K. Suwal, S. S. Choi, P. C. M. Planken, G. S. Park, N. K. Park, Q. H. Park, and D. S. Kim, Nat. Photon. 3, 152 (2009).

[31] J. R. Knab, A. J. L. Adam, M. Nagel, E. Shaner, M. A. Seo, D. S. Kim, and P. C. M. Planken, Opt. Express 17, 15072 (2009).

[32] A. O. Pinchuk and G. C. Schatz, Mater. Sci. Eng.: B 149, 251 (2008).

[33] S. Zou and G. C. Schatz, J. Chem. Phys. 121, 12606 (2004).

[34] S. Zou and G. C. Schatz, Chem. Phys. Lett. 403, 62 (2005).

[35] A. D. Humphrey and W. L. Barnes, Phys. Rev. B 90, 075404 (2014).

[36] C. Yan and O. J. F. Martin, Acs Nano 8, 11860 (2014). 